\title{
Effects and Mechanisms of Vitamin C Post-Conditioning on Platelet Activation after Hypoxia/Reoxygenation
}

\author{
Demin Liu Dongguo Pei Haijuan Hu Guoqiang Gu Wei Cui \\ Department of Cardiology, Second Hospital of Hebei Medical University, Shijiazhuang, PR China
}

\section{Keywords}

Vitamin C · Reactive oxygen species · Platelets · Apoptosis . Platelet aggregation

\begin{abstract}
Background: Platelet activation occurs upon ischemia/reperfusion and is related to the generation of reactive oxygen species (ROS) during this process. Vitamin C (VC) is a powerful antioxidant. VC scavenges ROS, reduces platelet activation, and attenuates reperfusion injury. However, the effects of VC on platelets undergoing hypoxia/reoxygenation $(H / R)$ remain unclear. Objectives: Herein, we evaluated the effects of $\mathrm{VC}$ on platelets in vitro following $\mathrm{H} / \mathrm{R}$ and the related mechanisms. Method: Fresh platelets were collected from 67 volunteers at the Blood Center of Hebei Province. Platelets were diluted with saline to a concentration of $2.00 \times$ $10^{11} / \mathrm{L}$. Aggregation and the curve slope were evaluated within $4 \mathrm{~h}$ with a whole-blood impedance analyzer. To determine the optimal experimental time, platelets were treated with hypoxia or reoxygenation for different times, and impedance aggregometry was carried out by measuring changes in electrical impedance induced by arachidonic acid $(0.5 \mathrm{~mm})$ and adenosine diphosphate $(10 \mu \mathrm{M})$, thereby establishing the $\mathrm{H} / \mathrm{R}$ model. Three antioxidants (VC, melatonin, and probucol) were used to treat platelets after $\mathrm{H} / \mathrm{R}$, and impedance aggregometry was used to determine their effects on platelet aggregation. The influence of VC on apoptosis-related indicators was detected. ROS and the mito-
\end{abstract}

\section{karger@karger.com} www.karger.com/tmh

Karger ${ }^{\prime \prime}=$
(C) 2019 The Author(s)

Published by S. Karger AG, Basel

This article is licensed under the Creative Commons AttributionNonCommercial-NoDerivatives 4.0 International License (CC BYNC-ND) (http//www.kargercom/Services/Open AccessLicense) UC-ND) (hip/No Usage and distribution for commercial purposes as well as any chondrial membrane potential were observed by inverted fluorescence microscopy and flow cytometry, respectively. Related protein levels were detected by Western blotting. Results: ROS scavengers inhibited platelet activation and aggregation in a concentration-dependent manner. VC post-conditioning scavenged ROS, downregulated cytochrome C, Bax, and caspase-9 proteins, and upregulated $\mathrm{BCl}-$ 2 protein. These effects collectively blocked platelet apoptosis and inhibited platelet aggregation. Conclusions: VC inhibited platelet aggregation by blocking apoptosis. Thus, VC may have applications in the treatment of platelet-related diseases.

(c) 2019 The Author(s)

Published by S. Karger AG, Basel

\section{Introduction}

In recent years, major advancements in medical treatments have improved blood flow to ischemic tissues and organs via reperfusion; however, secondary injury is caused by ischemic and anoxic/hypoxic tissue reperfusion (i.e., ischemia/reperfusion injury), and has become an important clinical issue. Many studies have shown that platelet activation plays a critical role in this process.

Platelets are cells derived from mature megakaryocytes in the bone marrow. These cells have no nucleus,

D.L. and D.P. contributed equally to this article. 
but have mitochondria and a plasma membrane. They also exhibit an irregular shape and function, primarily to maintain vascular integrity and prevent bleeding after vascular injury. In-depth studies of the physiological functions of platelets have revealed their key roles in human health and disease [1]. A study by Xu et al. [2] showed that activated platelets directly promoted the occurrence of reperfusion injury. Additionally, oxidative stress occurs during the reperfusion process of ischemic tissue, promoting the production of reactive oxygen species (ROS), triggering platelet activation, and causing secondary damage. Notably, the degree of platelet activation reflects the degree of myocardial ischemia. Ziegler et al. [3] ligated the left anterior descending arteries of the heart in mice for 0,20 , or $60 \mathrm{~min}$ to cause different degrees of myocardial ischemia, and then injected a new type of radioactive tracer combined with the activated GP IIb/IIIa molecule. Subsequent positron emission tomography of activated platelets to detect the extent of myocardial ischemia showed that this method was highly sensitive, particularly for patients who had undergone cardiac ischemia but had no significant changes in electrocardiograms or biomarkers.

Vitamin C (VC) is a natural water-soluble antioxidant with weak acidity and is involved in various antioxidant mechanisms in the human body. Notably, VC can inhibit platelet activation during ischemia/reperfusion [4], although the specific mechanisms are still unclear. Accordingly, in this study, we aimed to establish a hypoxia/reoxygenation $(\mathrm{H} / \mathrm{R})$ platelet model in vitro, observe the effects of VC post-treatment on platelet activity, and explore the specific mechanisms involved in VC-mediated changes in platelet activity to provide more theoretical evidence for its clinical use.

\section{Materials and Methods}

\section{Materials}

In this study, 67 platelet samples from healthy donors were obtained from Hebei Province Blood Center (Shijiazhuang, China). The platelet concentrations ranged from $9.60 \times 10^{11} / \mathrm{L}$ to $1.28 \times$ $10^{12} / \mathrm{L}$. Written informed consent was obtained from all donors, and the experimental procedures were approved by the Ethics Committee of the Second Hospital of Hebei Medical University (2017-R226). Samples included in this study were from blood donors who were strictly in accordance with the national blood donors' health examination standards. The blood platelet concentration was determined using a whole-blood cell analyzer (XE-Z100, Symex, Kobe, Japan). Platelets were then diluted to $2.00 \times 10^{11} / \mathrm{L}$ with saline. The experiment was completed within $4 \mathrm{~h}$ after the collection of platelets.

\section{Chemicals/Reagents}

Arachidonic acid (AA) and adenosine diphosphate (ADP) were supplied by Chrono-Log (Havertown, PA, USA). VC was procured from Sigma-Aldrich (St. Louis, MO, USA). Melatonin was from Biovision (Milpitas, San Francisco, CA, USA). Probucol, $2^{\prime}, 7^{\prime}$-dichlorodihydrofluorescein diacetate (DCFH-DA) and JC-1 were purchased from Cayman Chemical (Ann Arbor, MI, USA). Antibodies targeting cytochrome C, Bax, Bcl-2, caspase-9, and $\beta$-actin were provided by Affinity Biosciences (Cincinnati, $\mathrm{OH}$, USA). Dimethyl sulfoxide (DMSO) was purchased from Bosheng Chemical Reagents (Shijiazhuang, China). RIPA lysis buffer and phenylmethylsulfonyl fluoride (PMSF) storage solution were from Beijing Zhuang League International Biological Gene Technology (Beijing, China). All other reagents were of analytical grade.

\section{Establishment of the H/R Platelet Model in vitro}

Impedance aggregometry was carried out by measuring changes in electrical impedance using a Chrono-Log Aggregometer (model 590). One milliliter of diluted platelet protoplasm was added to the five incubators and placed in a hypoxic $\mathrm{CO}_{2}$ incubator $\left(\mathrm{N}_{2}\right.$ concentration: $94 \%, \mathrm{O}_{2}$ concentration: $1 \%, \mathrm{CO}_{2}$ concentration: 5\%). Samples were removed after 0, 5, 10, 20, and $30 \mathrm{~min}$. We found that $20 \mathrm{~min}$ was the optimal time for hypoxia. After hypoxia for $20 \mathrm{~min}$, reoxygenation was carried out for $0,1,3,5$, or 10 $\mathrm{min}$, and platelet aggregation was detected. The activation rate of platelet aggregation was calculated according to the following formula: platelet aggregation activation rate $(\%)=$ (platelet aggregation rate of experimental group $[\Omega] /$ control group of platelet aggregation rate $[\Omega]-1) \times 100$. We found that 5 min was the optimal time for reoxygenation.

Treatment of Platelets Subjected to $H / R$ with Antioxidants

For the preparation of VC, $1.7612 \mathrm{~g}$ of $\mathrm{VC}$ powder was dissolved in normal saline to $100 \mathrm{~mL}$, yielding a concentration of 100 $\mathrm{mM}\left(\mathrm{pH}\right.$ 2.37). This sample was stored at $4{ }^{\circ} \mathrm{C}$ and diluted to 10 , 100 , or $1,000 \mu \mathrm{M}$ for use.

Melatonin $(0.023228 \mathrm{~g})$ and probucol $(0.05168 \mathrm{~g})$ powders were dissolved separately in DMSO to $10 \mathrm{~mL}$, yielding concentrations of $10 \mathrm{mM}$. The solutions were stored at $-20^{\circ} \mathrm{C}$ in the dark and each diluted to $0.01,1$, or $100 \mu \mathrm{M}$ for use in experiments.

One milliliter of diluted platelets was added to the five incubators and placed in the hypoxic $\mathrm{CO}_{2}$ incubator as before for $20 \mathrm{~min}$. Next, the diluted platelets were incubated with various concentrations of VC, melatonin, or probucol for $5 \mathrm{~min}$, after which AA $(0.5$ $\mathrm{mM})$ or $\mathrm{ADP}(10 \mu \mathrm{M})$ was added. To evaluate the antiplatelet effects of these drugs, the percentage inhibition of platelet aggregation by various agents was determined.

\section{Detection of Platelet Apoptosis-Related Indexes following VC} Treatment

Determination of Endogenously Generated ROS

Endogenous ROS production in platelets was determined using DCFH-DA, an ROS-sensitive fluorescent probe. Untreated control and treated platelets were incubated with $10 \mu \mathrm{M}$ of DCFH-DA for $30 \mathrm{~min}$ at $37^{\circ} \mathrm{C}$. An inverted fluorescence microscope (Carl Zeiss AG, Oberkochen, Germany) was used to image samples at an excitation wavelength of $530 \mathrm{~nm}$.

\section{Determination of Changes in Mitochondrial Membrane}

Potential $(\Delta \psi \mathrm{m})$

A FACS Calibur flow cytometer (Becton Dickinson, San Jose, CA, USA) was used to analyze $\Delta \psi \mathrm{m}$ after the addition of $3 \mu \mathrm{L}$ of JC- 1 (final concentration $3 \mu \mathrm{g} / \mathrm{mL}$ ) to platelets. The platelets were then incubated at $37^{\circ} \mathrm{C}$ for $20 \mathrm{~min}$ in the dark. Green fluorescence, indicating combination with the JC-1 monomer, was used to evaluate the percentages of mitochondria-depolarized platelets. 


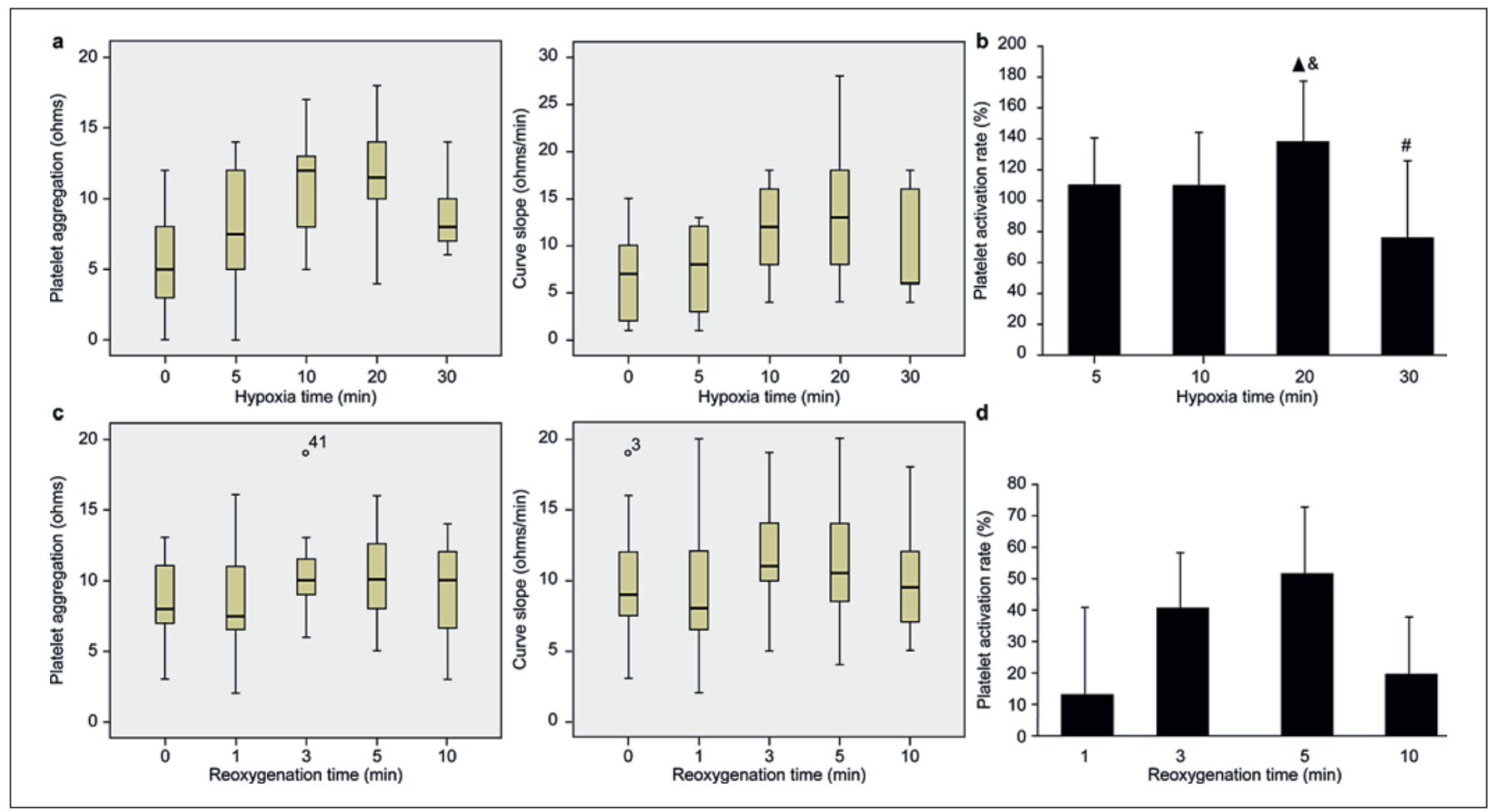

Fig. 1. Effects of hypoxia and reoxygenation for various times on platelet aggregation. a, b Compared with control platelets under atmospheric conditions, platelets exposed to hypoxia showed increased aggregation and curve slope, which reached a maximum after 20 min of hypoxia. c, d Compared with the 0 min group, platelet aggregation and the curve slope in other groups increased and the activation rate reached a maximum in the 5 min group, but no statistical difference was observed $(p>0.05)$. Data are presented as the mean \pm SD. ${ }^{\boldsymbol{\Delta}} p<0.05$ versus the 5 min group; ${ }^{\&} p<0.05$ versus the 10 min group; ${ }^{*} p<0.05$ versus the 20 min group.
Determination of Apoptosis-Related Proteins in Platelets

For the preparation of lysates, platelets were placed at a low temperature in a high-speed centrifuge $\left(8,000 \mathrm{~g}, 4^{\circ} \mathrm{C}\right.$ for $\left.10 \mathrm{~min}\right)$. The supernatant was then removed, leaving only a white precipitate, and $50 \mu \mathrm{L}$ of phosphate-buffered saline was added to an equal amount of platelet lysate (RIPA lysate: PMSF storage solution =100:1). The solution was then mixed and incubated on ice for $30 \mathrm{~min}$ for complete cell lysis. Lysates were centrifuged at $12,000 \mathrm{~g}$ for $10 \mathrm{~min}$, and supernatants were collected as total platelet protein samples.

For Western blot analyses, proteins were separated by sodium dodecyl sulfate polyacrylamide gel electrophoresis on $10 \%$ gels and transferred to polyvinylidene difluoride membranes. The membranes were blotted with specific antibodies against cytochrome $\mathrm{C}$ (1:1,000), Bax (1:1,000), Bcl-2 (1:1,000), and caspase-9 (1:1,000; all from Abcam, Cambridge, MA, USA) at $4{ }^{\circ} \mathrm{C}$ overnight and then incubated with goat anti-rabbit secondary antibodies $(1: 5,000)$ for $2 \mathrm{~h}$. The protein bands were detected using a Chemiluminescence Plus Western Blot Analysis Kit (Bio-Rad, Hercules, CA, USA). Relative quantification was carried out by measuring the band density with Quantity One V4.6.2 software (Bio-Rad). Bands of interest were normalized to the expression of $\beta$-actin. The experiments were replicated at least three times.

\section{Data Analysis}

All data are expressed as means \pm standard deviation. Statistical analysis was performed with SPSS 22.0 software (IBM, Chicago, IL, USA). Statistical significance among groups was determined by oneway analysis of variance followed by Tukey's test for comparison of means. Differences with $p$ values $<0.05$ were considered significant.

\section{Results}

\section{Effects of Hypoxia or Reoxygenation Time on Platelet} Aggregation

Compared with control platelets maintained under atmospheric conditions, platelets exposed to H/R showed an increased platelet aggregation and curve slope, with maximum responses after $20 \mathrm{~min}$ of hypoxia. Significant differences were observed between the 5 and $20 \mathrm{~min}$ groups $(p<0.05)$; however, there were no significant differences among the 5, 10, and 30 min groups $(p>0.05$; Fig. 1a, b).

In our analysis of reoxygenation time, platelet aggregation and curve slopes increased in all groups compared with the 0 min control group, and the activation rate reached a maximum in the 5 min group; however, there were no significant differences observed among all groups $(p>0.05$; Fig. 1c, d).

\section{Effect of Antioxidant Post-Treatment on Platelets Subjected to $H / R$}

Compared with the H/R group, platelet aggregation in cells treated with $1,000 \mu \mathrm{M}$ of VC was decreased significantly $(p<0.05)$. In contrast, no significant differences 


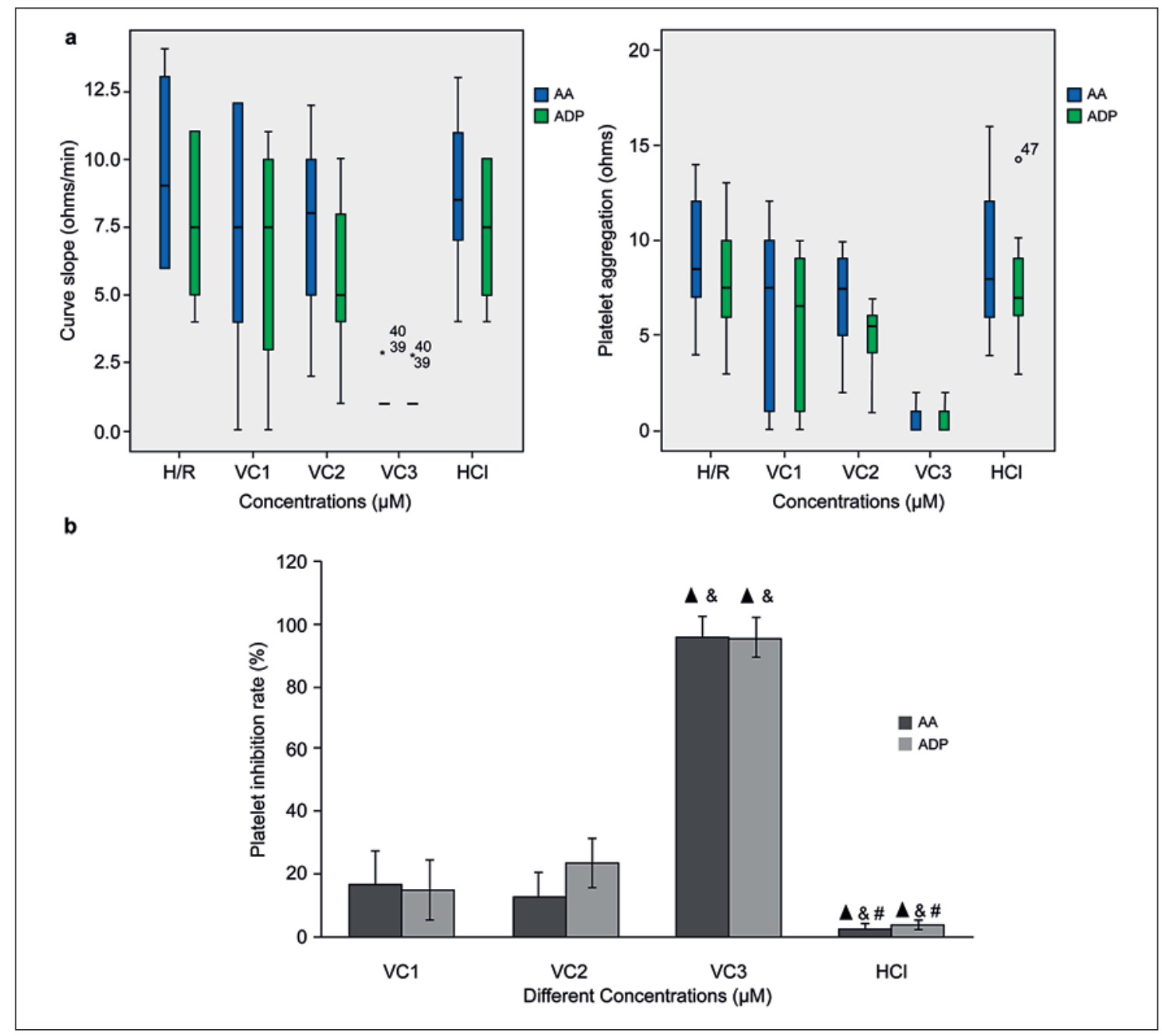

Fig. 2. Effects of different concentrations of VC on platelet aggregation and inhibition rates after hypoxia/reperfusion. a Compared with the hypoxia/reperfusion group, platelet aggregation in the VC3 group decreased significantly $(p<0.05)$, while no statistical difference was found for the VC1, VC2, and $\mathrm{HCl}$ groups $(p>0.05)$. b Compared with the hypoxia/reperfusion group, the platelet in- hibition rate in the VC3 group increased significantly $(p<0.05)$, while no statistical difference was found for the VC1 and VC2 groups $(p>0.05)$. Data are presented as the mean $\pm \mathrm{SD} .{ }^{\boldsymbol{\Delta}} p<0.05$ versus VC1; ${ }^{*} p<0.05$ versus $\mathrm{VC} 2{ }^{*}{ }^{\#} p<0.05$ versus VC3. VC1, vitamin C $10 \mu \mathrm{M}$; VC2, vitamin C $100 \mu \mathrm{M}$; VC3, vitamin C 1,000 $\mu \mathrm{M}$. were observed in the 10 and $100 \mu \mathrm{M}$ and $\mathrm{HCl}$ groups $(p>$ $0.05)$. Treatment with $1,000 \mu \mathrm{M}$ of VC showed the highest inhibition rate ( $p<0.05$; Fig. 2$)$.

Notably, platelet aggregation in cells treated with 100 $\mu \mathrm{M}$ melatonin was decreased significantly $(p<0.05) \mathrm{com}-$ pared with the H/R group. There were no significant differences in the 0.01 and $1 \mu \mathrm{M}$ groups $(p>0.05)$. Moreover, the $100 \mu \mathrm{M}$ group showed the highest inhibition rate $(p<$ 0.05 ), and $0.01 \mu \mathrm{M}$ melatonin activated, but did not inhibit, platelet aggregation (Fig. 3).

Finally, we analyzed the effects of probucol on platelet aggregation. Compared with the H/R group, platelet aggregation in the probucol group $(100 \mu \mathrm{M})$ decreased significantly $(p<0.05)$, whereas no significant differences were found in the 1 and $10 \mu \mathrm{M}$ groups $(p>0.05)$. Again, the $100 \mu \mathrm{M}$ group showed the highest inhibition rate compared with the 1 and $10 \mu \mathrm{M}$ groups $(p<0.05$; Fig. 4).

Effects of VC on Platelet Apoptosis-Related Indicators

Compared with the control group, ROS levels were significantly increased in the $\mathrm{H} / \mathrm{R}, \mathrm{VC}$, and $\mathrm{HCl}$ groups. Moreover, ROS levels in the VC group decreased, whereas those in the $\mathrm{HCl}$ group showed no changes compared with ROS levels in the H/R group. Thus, these findings suggested that VC acted as a powerful ROS scavenger (Fig. 5a).

Next, we evaluated the $\Delta \psi \mathrm{m}$. Compared with the control group, $\Delta \psi \mathrm{m}$ values in the $\mathrm{H} / \mathrm{R}, \mathrm{VC}$, and $\mathrm{HCl}$ groups increased significantly $(p<0.05)$. Additionally, $\Delta \psi \mathrm{m}$ values in the VC group decreased compared with those in the H/R group $(p<0.05)$, and there were no 


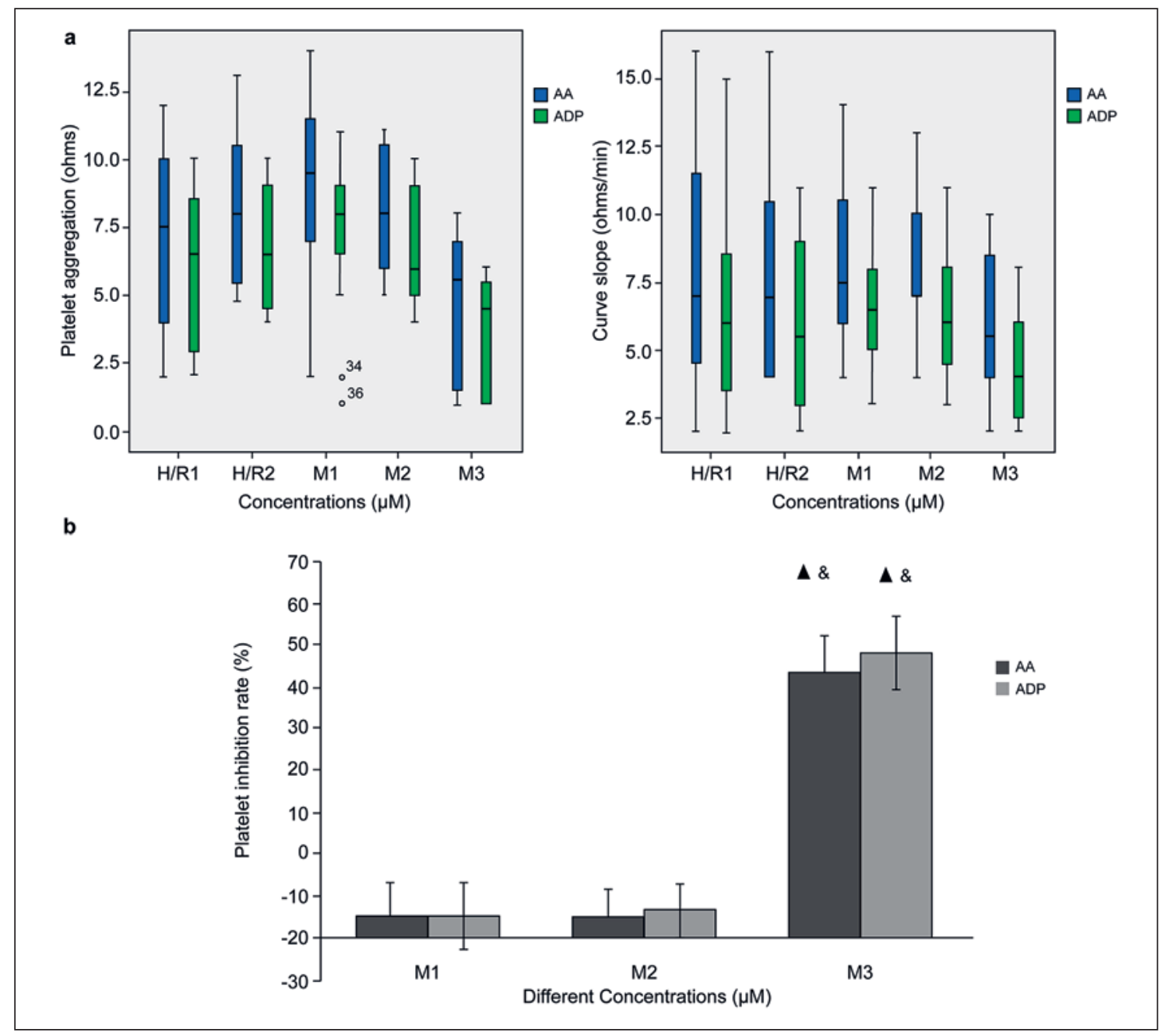

Fig. 3. Effects of different concentrations of melatonin on platelet aggregation and inhibition rates after hypoxia/reperfusion. a Compared with the hypoxia/reperfusion group, platelet aggregation in the M3 group decreased significantly $(p<0.05)$, while no statistical difference was found for the M1 and M2 groups ( $p>$ 0.05). $\mathbf{b}$ Compared to the M1 and M2 groups, the M3 group showed the greatest inhibition rate $(p<0.05)$. Data are presented as the mean \pm SD. ${ }^{\wedge} p<0.05$ versus M1; ${ }^{\&} p<0.05$ versus M2. H/R1, 10 $\mu \mathrm{L} 0.9 \%$ sodium chloride injection solution; H/R2, $10 \mu \mathrm{L}$ DMSO; M1, melatonin $0.01 \mu \mathrm{M}$; M2, melatonin $1 \mu \mathrm{M}$; M3, melatonin 100 $\mu \mathrm{M}$. significant differences $(p>0.05)$ between the $\mathrm{HCl}$ and $\mathrm{H} / \mathrm{R}$ groups, suggesting that the ability to alleviate mitochondrial injury was related to ROS scavenging, not acidity (Fig. 5b).

Finally, we analyzed changes in the expression of apoptosis-related proteins, including Bax, Bcl-2, cytochrome $\mathrm{C}$, and caspase-9. Our results showed that the levels of cytochrome C, Bax, and caspase- 9 increased in the experimental groups compared with those in the control group, suggesting that cytoplasmic apoptotic events related to the mitochondrial pathway were stimulated in platelets. Notably, in the VC group, the expression levels of cytochrome C, Bax, and caspase- 9 decreased, whereas the expression level of Bcl-2 increased significantly compared with the H/R group. These results demonstrated that VC suppressed activation of the hypoxia-induced intrinsic apoptosis pathway and hindered AA-induced platelet aggregation and adhesion (Fig. 5c, d).

\section{Discussion}

Ischemia/reperfusion injury involves many pathophysiological processes, including platelet activation [5]. The process of organ/tissue ischemia/reperfusion can cause the production and release of active oxygen in nucleated cells [6]. Moreover, platelets can produce ROS after various physiological or pathological stimuli, and the formation of thrombi is accompanied by the release of ROS $[7,8]$. ROS are involved in platelet formation, activation, or enzyme digestion. In recent years, studies have 


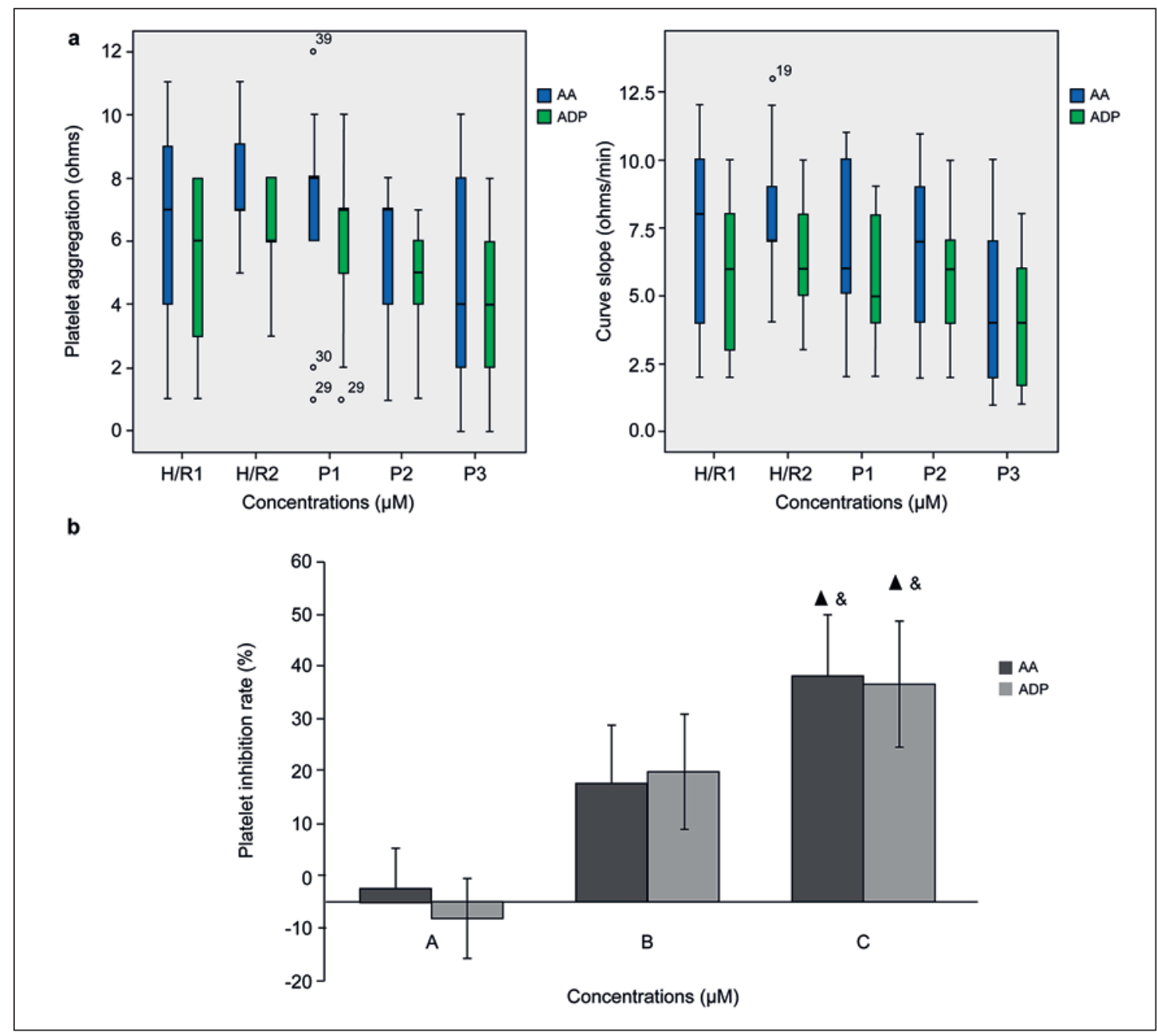

Fig. 4. Effects of different concentrations of probucol on platelet aggregation and inhibition rates after hypoxia/reperfusion. a Compared to the hypoxia/reperfusion group, platelet aggregation in the $\mathrm{P} 3$ group decreased significantly $(p<0.05)$, while no statistical difference was found for the P1 and P2 groups $(p>0.05)$. b Compared to the P1 and P2 groups, the P3 group showed the greatest inhibition rate $(p<0.05)$. Data are presented as the mean \pm SD. $\boldsymbol{\Delta} p<0.05$ versus $\mathrm{P} 1 ;{ }^{\&} p<0.05$ versus P2. H/R $1,10 \mu \mathrm{L} 0.9 \%$ sodium chloride injection solution; H/R2, $10 \mu \mathrm{L}$ DMSO; P1, probucol $1 \mu \mathrm{M}$; P2, probucol $10 \mu \mathrm{M}$; P3, probucol $100 \mu \mathrm{M}$. shown that ROS in platelets are derived from the NADH/ $\mathrm{NADPH}$ oxidase system $[9,10]$ and the metabolism of AA; the former is the main source of ROS and plays a major role in determining the levels of ROS in platelets.

Platelet apoptosis is a major pathway for platelet death [11]. Similar to other cells, platelet homeostasis is the result of a balance between platelet production and destruction. Although platelets are nucleus-free blood cells, they contain many apoptotic and anti-apoptotic proteins, and ribosomes from megakaryocytes permit protein synthesis in platelets [12]. Many factors can induce apoptosis in platelets, including high blood flow shear force [13], thrombin [14], collagen [7], the calcium ion carrier A23187, and platelet storage conditions [15]. Additionally, ROS play very important roles in thrombocytopenia and collagen-induced apoptosis [16]. Application of an- tioxidants can inhibit apoptosis [17], and thrombin and collagen induce platelet activation and aggregation, suggesting that there is a correlation between platelet apoptosis and activation [18].

Previous studies have shown that apoptotic platelets produce particles that subsequently promote platelet activation and accelerate platelet aggregation $[19,20]$. To eliminate the interference of a variety of confounding factors in the body, we simulated ischemia/reperfusion in an isolated platelet $\mathrm{H} / \mathrm{R}$ model $[21,22]$. We then selected three antioxidants (VC, melatonin, and probucol) to observe the effects of antioxidants on platelet aggregation and to detect apoptosis-related indexes in platelets in order to explore the specific mechanisms through which $\mathrm{VC}$ affects $\mathrm{H} / \mathrm{R}$ in platelets. Our results showed that the three antioxidants inhibited platelet activation to varying 

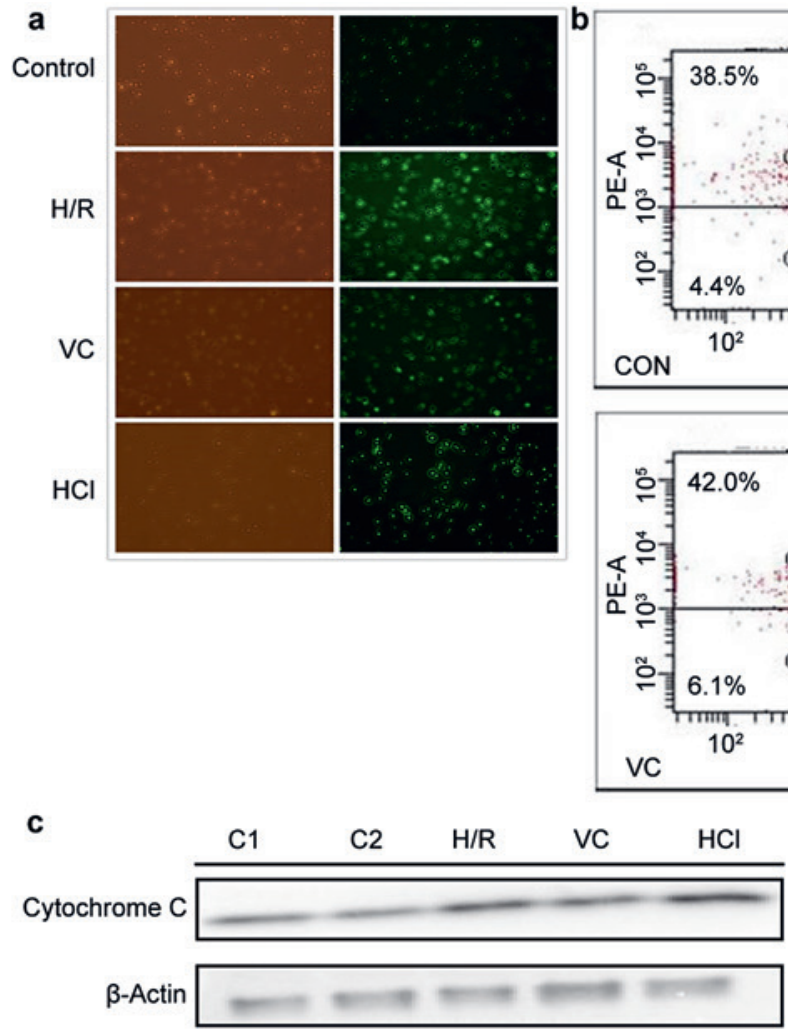
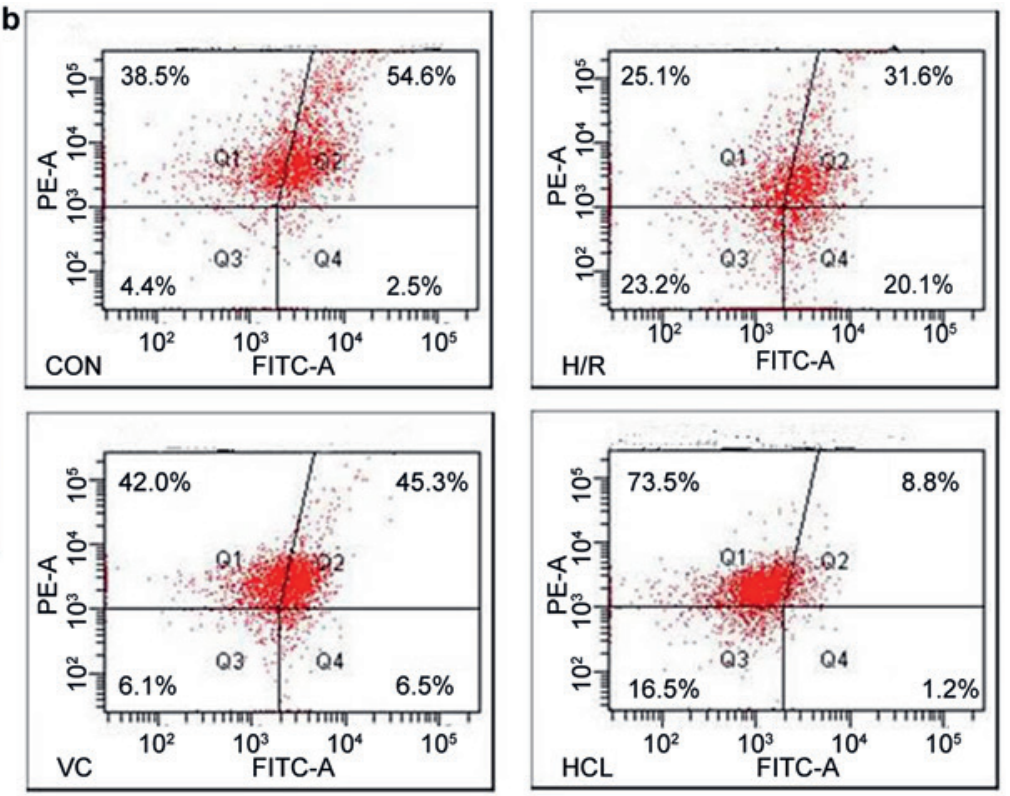

d $\quad$ C1

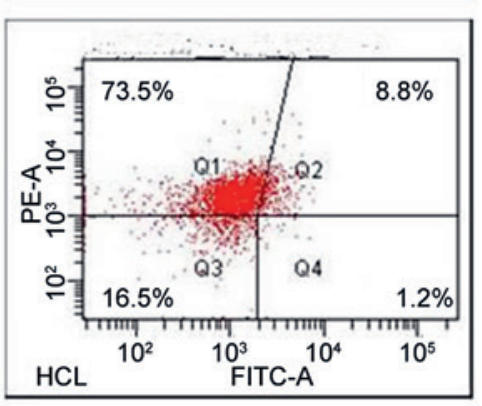

C2
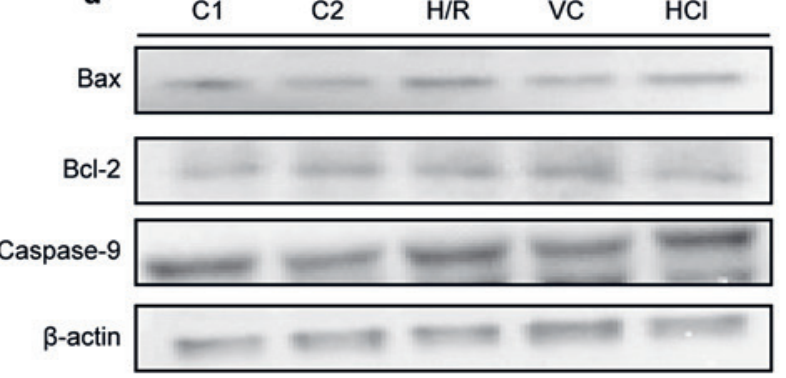

Fig. 5. Effects of VC on platelet apoptosis-related indicators. a ROS generation in the different groups, as observed by inverted fluorescence microscopy $(\times 10)$. Compared with the control group, ROS levels significantly increased in the $\mathrm{H} / \mathrm{R}, \mathrm{VC}$, and $\mathrm{HCl}$ groups. Compared with the H/R group, ROS levels in the VC group significantly decreased $(p<0.05)$. b Mitochondrial membrane potential in the different groups, as determined by flow cytometry. $\mathrm{Mi}$ tochondrial membrane potential $(\Delta \psi \mathrm{m})$ values in the VC group decreased compared with those in the H/R group $(p<0.05)$. c In the VC group, the expression level of cytochrome $\mathrm{C}$ decreased significantly compared with the H/R group $(p<0.05)$. d The expression levels of Bax and caspase- 9 decreased in the VC group, whereas the expression of Bcl-2 increased significantly compared with the H/R group $(p<0.05)$. H/R, hypoxia/reperfusion; VC, vitamin $\mathrm{C} 1,000 \mu \mathrm{M} ; \mathrm{HCl}, 10 \mu \mathrm{L}$ hydrochloric acid ( $\mathrm{pH}=2.37$ ); $\mathrm{C}$, platelets at room temperature; $\mathrm{C} 2$, platelets at $37^{\circ} \mathrm{C}$ in order to exclude the influence of temperature. degrees in concentration-dependent manners. Additionally, ROS produced by H/R increased, and the activation degree increased, while antioxidants removed ROS and inhibited the activation and further aggregation of blood platelets.

$\mathrm{VC}$, also known as L-ascorbic acid, is a natural watersoluble, weakly acidic vitamin with strong antioxidant ability [23]. Recent studies have reported the antiplatelet effects of VC [24]. In this study, we confirmed that high concentrations of VC strongly inhibited platelet aggregation, whereas the $\mathrm{HCl}$ group did not show obvious inhibitory effects. Additionally, inverted fluorescence microscopy showed that ROS levels were significantly lower in the VC group than in the H/R group, indicating that acidity was not related to platelet aggregation effects.

Melatonin is an indole neuroendocrine hormone secreted by the pineal body. Many basic and clinical studies have confirmed that melatonin has extensive effects on biological rhythm and on the reproductive, immune, digestive, central nervous, and cardiovascular systems [25]. Moreover, melatonin has antioxidant and antitumor effects and is widely used for immunomodulation, antiaging, and antitumor treatments [26]. Importantly, in this study, we found that low concentrations of melatonin activated platelet aggregation, whereas higher concentra- 
tions inhibited platelet aggregation, similar to the results of previous studies [27].

Probucol was used in the 1970s in the USA and was believed to reduce serum cholesterol. Recent studies have shown that probucol also has antiatherosclerosis effects, modulates oxidative stress, and protects the blood vessel endothelium [28]. During exposure to hypoxia, platelets produce large amounts of ROS, and active oxygen can induce aggregation by participating in platelet-related signal transduction pathways [29]. Moreover, antioxidants can inhibit platelet aggregation by removing the produced ROS. In this study, we showed that probucol inhibited platelet activation and aggregation in a concentration-dependent manner. Thus, our results showed that all three antioxidants inhibited the aggregation of platelets exposed to $\mathrm{H} / \mathrm{R}$, indicating that activation and aggregation of platelets exposed to $H / R$ were closely related to oxidative stress.

$\mathrm{VC}$ is not widely used in the clinical setting during ischemia/reperfusion therapy. In this study, we confirmed that VC post-conditioning inhibited platelet activation during $\mathrm{H} / \mathrm{R}$. The decreased ROS and platelet apoptosis index in the VC group supported the relationship between inhibition of platelet aggregation and anti-apoptotic effects. Moreover, the results of the $\mathrm{HCl}$ group (at the same $\mathrm{pH}$ ) were not significantly different from those of the H/R group, indicating that the inhibitory effects on platelet aggregation were not related to the weak acidity of the treatment.

As described above, platelet apoptosis is a type of programmed cell death induced by various physiological and pathological stimuli. Apoptosis induced in platelets occurs mainly through signaling pathways in the mitochondria; these signaling pathways affect the function of the mitochondria, decrease the $\Delta \psi \mathrm{m}$, and activate apoptotic proteins, resulting in activation of caspase- 3 and induction of apoptosis [30]. During this process, mitochondria provide energy for cell survival and act as the center of apoptosis regulation. Proapoptotic Bax and antiapoptotic Bcl-2 play important roles in the regulation of platelet apoptosis, and the relative ratio of these proteins determines whether cells undergo programmed cell death. In this study, we found that H/R significantly increased the $\Delta \psi \mathrm{m}$ and cytochrome C, caspase- 9 , and Bax expression, and significantly decreased Bcl-2 expression, indicating that platelet apoptosis played an important role in platelet activation. When $\mathrm{VC}$ was added before reoxygenation, the $\Delta \psi \mathrm{m}$ and cytochrome $\mathrm{C}$, caspase- 9 , and Bax expression decreased, whereas Bcl-2 expression increased. Thus, these results showed that VC inhibited platelet aggregation by blocking apoptosis. Moreover, these effects were related to ROS scavenging ability, not acidity.

In clinical trials, Basili et al. [31] found that VC treatment inhibited platelet activation to some extent, consis- tent with the results of the present study. This suggests that VC could be used as an antiplatelet drug. Indeed, we propose that VC could be applied to reduce the extent of tissue and organ ischemia/reperfusion injury. However, further studies are needed to assess these effects, particularly in patients with various comorbidities.

This study had some limitations. First, the use of an isolated platelet $\mathrm{H} / \mathrm{R}$ model cannot accurately assess the effects of leukocytes, macrophages, and various cytokines in the body, and the drug may interact with other drugs in the body. Therefore, the effects of VC on platelet aggregation are expected to be more complex in vivo. In addition, platelet collection and experimental procedures may lead to partial platelet activation, affecting the accuracy of the results.

\section{Conclusion}

We found that ROS scavengers concentration-dependently inhibited platelet activation and aggregation. Moreover, VC post-conditioning scavenged ROS and caused downregulation of cytochrome C, Bax, and caspase- 9 proteins and upregulation of the Bcl-2 protein. VC treatment also reduced platelet apoptosis and inhibited platelet aggregation.

\section{Statement of Ethics}

Written informed consent was obtained from all blood donors, and the experimental procedures were approved by the Ethics Committee of the Second Hospital of Hebei Medical University (2017-R226).

\section{Disclosure Statement}

The authors have no conflicts of interest to declare.

\section{Funding Sources}

This work was supported by the National Natural Science Foundation of China (No. 81402510) and Hebei Province Natural Science Foundation (No. H2015206139).

\section{Author Contributions}

W.C. was involved in study design and manuscript editing; D.L. and D.P. performed the majority of the laboratory work and contributed to writing the manuscript; H.H. and G.G. were responsible for the analyses of data. 


\section{References}

1 Ferroni P, Vazzana N, Riondino S, Cuccurullo C, Guadagni F, Davì G. Platelet function in health and disease: from molecular mechanisms, redox considerations to novel therapeutic opportunities. Antioxid Redox Signal. 2012 Nov; 17(10):1447-85.

$2 \mathrm{Xu}$ Y, Huo Y, Toufektsian MC, Ramos SI, Ma $\mathrm{Y}$, Tejani AD, et al. Activated platelets contribute importantly to myocardial reperfusion injury. Am J Physiol Heart Circ Physiol. 2006 Feb;290(2):H692-9.

3 Ziegler M, Alt K, Paterson BM, Kanellakis P, Bobik A, Donnelly PS, et al. Highly sensitive detection of minimal cardiac ischemia using positron emission tomography imaging of activated platelets. Sci Rep. 2016 Dec;6(1): 38161-70.

4 Pignatelli P, Tanzilli G, Carnevale R, Di Santo S, Loffredo L, Celestini A, et al. Ascorbic acid infusion blunts CD40L upregulation in patients undergoing coronary stent. Cardiovasc Ther. 2011 Dec;29(6):385-94

5 Gawaz M. Role of platelets in coronary thrombosis and reperfusion of ischemic myocardium. Cardiovasc Res. 2004 Feb;61(3): 498-511.

6 Bagheri F, Khori V, Alizadeh AM, Khalighfard S, Khodayari S, Khodayari H. Reactive oxygen species-mediated cardiac-reperfusion injury: mechanisms and therapies. Life Sci. 2016 Nov; 165:43-55.

7 Caccese D, Praticò D, Ghiselli A, Natoli S, Pignatelli $\mathrm{P}$, Sanguigni V, et al. Superoxide anion and hydroxyl radical release by collagen-induced platelet aggregation-role of arachidonic acid metabolism. Thromb Haemost. 2000 Mar;83(3):485-90.

8 Wachowicz B, Olas B, Zbikowska HM, Buczyński A. Generation of reactive oxygen species in blood platelets. Platelets. 2002 May; 13(3):175-82.

9 Krötz F, Sohn HY, Gloe T, Zahler S, Riexinger $\mathrm{T}$, Schiele TM, et al. NAD $(\mathrm{P}) \mathrm{H}$ oxidase-dependent platelet superoxide anion release increases platelet recruitment. Blood. 2002 Aug; 100(3):917-24.

10 Delaney MK, Kim K, Estevez B, Xu Z, Stojanovic-Terpo A, Shen B, et al. Differential roles of the NADPH-oxidase 1 and 2 in platelet activation and thrombosis. Arterioscler Thromb Vasc Biol. 2016 May;36(5):846-54.
11 Lebois M, Josefsson EC. Regulation of platelet lifespan by apoptosis. Platelets. 2016 Sep; 27(6):497-504.

12 Gyulkhandanyan AV, Mutlu A, Freedman J, Leytin V. Markers of platelet apoptosis: methodology and applications. J Thromb Thrombolysis. 2012 May;33(4):397-411.

13 Leytin V, Allen DJ, Mykhaylov S, Mis L, Lyubimov EV, Garvey B, et al. Pathologic high shear stress induces apoptosis events in human platelets. Biochem Biophys Res Commun. 2004 Jul;320(2):303-10.

14 Leytin V, Allen DJ, Mykhaylov S, Lyubimov E, Freedman J. Thrombin-triggered platelet apoptosis. J Thromb Haemost. 2006 Dec; 4(12):2656-63.

15 Leytin V, Freedman J. Platelet apoptosis in stored platelet concentrates and other models. Transfus Apheresis Sci. 2003 Jun;28(3): 285-95.

16 Wang X, Zhang P, Zhao L, Tu Y, Dai K. [Study of reactive oxygen species on the regulation of platelet apoptosis]. Zhonghua Xue Ye Xue Za Zhi. 2014 Jun;35(6):511-4. Chinese.

17 Thushara RM, Hemshekhar M, Santhosh MS, Jnaneshwari S, Nayaka SC, Naveen S, et al. Crocin, a dietary additive protects platelets from oxidative stress-induced apoptosis and inhibits platelet aggregation. Mol Cell Biochem. 2013 Jan;373(1-2):73-83.

18 Lopez JJ, Salido GM, Pariente JA, Rosado JA. Thrombin induces activation and translocation of Bid, Bax and Bak to the mitochondria in human platelets. J Thromb Haemost. 2008 Oct;6(10):1780-8.

19 Morel O, Jesel L, Freyssinet JM, Toti F. Cellular mechanisms underlying the formation of circulating microparticles. Arterioscler Thromb Vasc Biol. 2011 Jan;31(1):15-26.

20 Mallat Z, Benamer H, Hugel B, Benessiano J, Steg PG, Freyssinet JM, et al. Elevated levels of shed membrane microparticles with procoagulant potential in the peripheral circulating blood of patients with acute coronary syndromes. Circulation. 2000 Feb;101(8):841-3.

21 Pönicke K, Sternitzky R, Mest HJ. Stimulation of aggregation and thromboxane A2 formation of human platelets by hypoxia. Prostaglandins Leukot Med. 1987 Sep;29(1):49-59.
22 Iuliano L, Colavita AR, Leo R, Praticò D, Violi F. Oxygen free radicals and platelet activation. Free Radic Biol Med. 1997;22(6):9991006.

23 Morel O, Jesel L, Hugel B, Douchet MP, Zupan $\mathrm{M}$, Chauvin $\mathrm{M}$, et al. Protective effects of vitamin $\mathrm{C}$ on endothelium damage and platelet activation during myocardial infarction in patients with sustained generation of circulating microparticles. J Thromb Haemost. 2003 Jan;1(1):171-7.

24 Olas B, Wachowicz B. Resveratrol and vitamin $\mathrm{C}$ as antioxidants in blood platelets. Thromb Res. 2002 Apr;106(2):143-8.

25 Pandi-Perumal SR, BaHammam AS, Ojike NI, Akinseye OA, Kendzerska T, Buttoo K, et al. Melatonin and human cardiovascular disease. J Cardiovasc Pharmacol Ther. 2017 Mar; 22(2):122-32

26 Tordjman S, Chokron S, Delorme R, Charrier A, Bellissant E, Jaafari N, et al. Melatonin: pharmacology, functions and therapeutic benefits. Curr Neuropharmacol. 2017 Apr; 15(3):434-43

27 Kumari S, Dash D. Melatonin elevates intracellular free calcium in human platelets by inositol 1,4,5-trisphosphate independent mechanism. FEBS Lett. 2011 Jul;585(14): 2345-51.

28 Li T, Chen W, An F, Tian H, Zhang J, Peng J, et al. Probucol attenuates inflammation and increases stability of vulnerable atherosclerotic plaques in rabbits. Tohoku J Exp Med. 2011 Sep;225(1):23-34.

29 Forde RC, Fitzgerald DJ. Reactive oxygen species and platelet activation in reperfusion injury. Circulation. 1997 Feb;95(4):787-9.

30 Rong Y, Distelhorst CW. Bcl-2 protein family members: versatile regulators of calcium signaling in cell survival and apoptosis. Annu Rev Physiol. 2008;70(1):73-91.

31 Basili S, Pignatelli P, Tanzilli G, Mangieri E, Carnevale R, Nocella C, et al. Anoxia-reoxygenation enhances platelet thromboxane A2 production via reactive oxygen species-generated NOX2: effect in patients undergoing elective percutaneous coronary intervention. Arterioscler Thromb Vasc Biol. 2011 Aug; 31(8):1766-71. 